\title{
Prevention of the Formation of Unaesthetic Scars Following the Surgical Cutaneous Wounds Healing of the Jaw and Facial Region Based on Transplantation of Xenogenic Fibroblasts
}

\author{
Marina N. Morozova, PhD, ScD; Alexey G. Baranovsky, PhD; \\ Yelena Yu. Shapovalova, PhD, ScD*; Svetlana A. Demyanenko, PhD, ScD; \\ Yuri G. Baranovsky, PhD; Tatiana A. Boyko, PhD; Fedor N. Ilchenko, PhD, ScD \\ Medical Academy named after S.I. Georgievsky of Vernadsky CFU \\ Simferopol, the Russian Federation
}

\begin{abstract}
The purpose of this study was to optimize healing and prevent the formation of unaesthetic scars of the maxillofacial region by transplanting xenogenic dermal fibroblasts (XDFs) into a fresh surgical wound.

Materials and Methods: A total of 26 patients were selected with formations located on the skin of the face and neck, which could be compared with symmetrical areas of the healthy side. The patients were divided into 2 groups - the main group (Group 1) and the control group (Group 2). In Group 2 ( $\mathrm{n}=12$ ), a neoplasm was dissected, followed by grafting with local tissues, and in the Group $1(\mathrm{n}=14)$, a culture suspension of XDFs was injected by a tunnel method into the wound edges before stitching. The area of scar formation was determined using the LesionMeter program for the Android operating system. Contact thermography was carried out using thermo indicator films (CelluVision kit, IPS Italy).

Results: The analysis of the parameters of the young scar formation on Day 30 after the operation makes it possible to state that the introduction of cell culture of XDFs in the wound edges has a positive effect on the healing rate of surgical wounds, decreases the inflammatory response and contributes to the development of a distinctively positive scar in terms of its quality and functional characteristics. By Day 30, the primary surgical wound area was reduced by $67.69 \%$, and $71.43 \%$ of patients had soft and thin aesthetic scars with microcirculation that were not distinguishable from the surrounding skin and the skin of symmetrical areas of the face or neck. In patients of the control group, without fibroblast transplantation, the area decreased by only $50.0 \%$ and aesthetic scars were formed only in $41.67 \%$ of cases. In $16.67 \%$ of patients, the presence of wide, dense, cold (due to weak vascularization) hypotrophic scars was noted. Hyperthermia persists around these scars, indicating a weak inflammatory response

Conclusion: The use of a cell culture suspension of XDFs in the treatment of postoperative surgical wounds opens up new real possibilities for reducing the incidence of inflammatory reactions, stimulates healing processes and contributes to the development of more functionally and aesthetically acceptable scars on the face and neck.(International Journal of Biomedicine. 2019;9(3):251-256.)
\end{abstract}

Key Words: surgical wound $\bullet$ fibroblasts $\bullet$ scar $\bullet$ planimetry $\bullet$ thermometry

\section{Introduction}

Surgeons specializing in surgery of the head and neck area deal with surgical wounds that are made with a sharp scalpel. They are characterized as incised wounds and at first glance have

*Corresponding author: Prof. Yelena Shapovalova, PhD, ScD. Head of the Department of Histology \& Embryology of the Medical Academy named after S.I. Georgievsky of Vernadsky CFU. Simferopol, Russia.E-mail: Shapovalova_L@mail.ru favorable conditions for healing. ${ }^{(1)}$ In most cases, surgical sutures make it possible to bring the wound edges together by no less than $5 \mathrm{~mm}$; then, due to swelling and reduction of the fibrin clot, it is possible to glue the wound edges.(2) Such wounds should heal quickly by primary intention and form a normotrophic, delicate, aesthetic skin scar. ${ }^{(3)}$ However, the use of precision operating techniques and suitable suture material does not guarantee against the development of pathological non-aesthetic scars, which patients perceive as unattractive. ${ }^{(4)}$ Due to various types of symmetry disorders, facial expressions or forms, scars on the face 
can cause not only discomfort, but also a decrease in a person's social adaptation. ${ }^{(5)}$ The presence of cicatricial changes on the face is especially hard on young people. ${ }^{(6)}$ More often than others, this cohort of patients seeks clinical help. ${ }^{(7)}$

The decisive link in wound healing is the main cells of the connective tissue - fibroblasts. They synthesize and release into the environment numerous biologically active substances: various growth factors, components of the extracellular matrix, and enzymes. With the help of which, collagen and hyaluronic acid are destroyed, and then these molecules are newly synthesized and a scar is formed. ${ }^{(8)}$ All of the modern methods of preventing the formation of pathological scars after surgery cannot fundamentally affect the function of fibroblasts; therefore, many methods of prevention and treatment have been proposed. ${ }^{(9)}$ One of the promising modern methods of accelerating the regeneration of fresh surgical wounds and the prevention of pathological scarring is the introduction into the wound area of various cultures of embryonic fibroblasts ${ }^{(10)}$ and mesenchymal stem cells (MSCs). ${ }^{(11)}$ In the case of embryonic fibroblasts, they enhance wound healing without scarring in the prenatal period in mammals and humans; ${ }^{(12)}$ in the case of MSCs, they introduce the possibility of differentiating into different cells of the regenerative cascade. ${ }^{(13)}$ The possibility of transplanting xenogenic fibroblasts, as a key factor of reparative regeneration, into a fresh operative wound of the scalp or neck in order to accelerate healing and prevent pathological scarring is relevant and has not received adequate coverage in clinical studies.

The purpose of this study was to optimize healing and prevent the formation of unaesthetic scars of the maxillofacial region by transplanting xenogenic dermal fibroblasts (XDFs) into a fresh surgical wound.

\section{Materials and Methods}

All studies were approved by the Ethics Committee of Medical Academy n.a. S.I. Georgievsky of Vernadsky CFU (Protocol No 6 dated May 10, 2018). Written informed consent was obtained from all participants.

\section{Clinical study}

For the study, patients were selected with formations located on the skin of the face and neck, which could be compared with symmetrical areas of the healthy side. A total of 26 patients ( 17 women and 9 men) aged between 20 and 52 years participated in the study (Table 1).

Table 1.

Main characteristics of the studied patients

\begin{tabular}{|l|c|c|c|}
\hline \multirow{2}{*}{ Clinical diagnosis } & \multicolumn{2}{|c|}{ Gender (n /\%) } & \multirow{2}{*}{ Average age } \\
\cline { 2 - 3 } & $\mathrm{M}$ & $\mathrm{F}$ & \\
\hline Nevus & $4 / 15.4$ & $1 / 3.8$ & $39.6 \pm 1.4$ \\
\hline Fibroma & $7 / 26.9$ & $5 / 19.2$ & $48.4 \pm 1.3$ \\
\hline Lipoma & $6 / 23.1$ & $3 / 11.5$ & $28.5 \pm 1.1$ \\
\hline
\end{tabular}

The patients were divided into 2 groups - the main group (Group 1) and the control group (Group 2). In Group 2 $(n=12)$, a neoplasm was dissected, followed by grafting with local tissues, and in the Group $1(\mathrm{n}=14)$, a culture suspension of XDFs was injected into the wound edges with a needle $30 \mathrm{G}$, $13 \mathrm{~mm}$ before stitching. Suspension of XDFs was used on the basis of an approved medical technology (FS No. 2009/398 dated July 21, 2010). The suspension consisted of growth medium alpha MEM (Lonza) and xenogenic fibroblasts. Depending on the size of the wound, 5 to 6 million cells were introduced. The skin formations were excised, taking into account the location of the Langer lines within healthy tissues. The edges of the wound before stitching were mobilized for drawn together without tension. The wound edges were fixed with an intradermal suture with atraumatic suture material (polypropylene, 5/0 and 4/0, depending on localization), which was removed on Day 7 after the operation.

\section{Cultivation and immunophenotyping of XDFs}

Dermal fibroblasts from the skin of young donors, obtained by plastic surgery, were enzymatically isolated under sterile boxing conditions with laminar airflow. After the enzymatic removal of the epidermis, the pieces of skin were placed on Alpha MEM (Lonza) and crushed with vascular scissors to a size of 1-2 mm. Then equal volumes of collagenase type I (200 U/ml, Sigma) and dispase (30 U/ml, Gibco) were added to the tissue pieces. The resulting mixture was incubated for 1 hour at $37^{\circ} \mathrm{C}$ with constant stirring. After filtering the suspension through a mesh filter with a diameter of $0.40 \mu \mathrm{m}$ and centrifugation for 7 minutes at $1000 \mathrm{rpm}$, fibroblasts were resuspended and cultured in Alpha MEM (Lonza) with the addition of $10 \%$ patient serum and 50 units $/ \mathrm{ml}$ penicillinstreptomycin (PanEco) in Petri dishes in an incubator at $37^{\circ} \mathrm{C}$ and a $\mathrm{CO}_{2}$ concentration of $5 \%$ to reach $100 \%$ confluent. For cell seeding, $0.25 \%$ trypsin- $0.02 \%$ EDTA was used.

The phenotype of populations of dermal fibroblasts was determined by means of the reaction of indirect immunofluorescence, using monoclonal antibodies with fluorescent labels. At the bottom of the Petri dishes, coverslips were placed, on which dermal fibroblasts were attached. Coverslips were removed upon reaching the confluent of the first passage. Cells were fixed with ice-cold acetone. For immunophenotyping of dermal fibroblasts, a panel of primary monoclonal antibodies to surface antigens was used: CD 34 [Clone EP373Y] (Abcam), CD 45 (Abcam), CD44 [Clone EPR1013Y] (Abcam), CD73 [Clone 7G2] (Abcam), CD90 (Thy -1) [Clone 5E10] (BD Biosciences), CD31 [Clone 1D12] (Cell Marque), CD 105 (Endoglin) [Clone EPR10145] (Abcam). Secondary antibodies were conjugated with Alexa Fluor 488 and Alexa Fluor 594 fluorescent labels (Invitrogen). The preparations were studied using an Olympus BX-43 fluorescence microscope.

For the introduction into the fresh surgical wound of patients of Group 1, XDFs with the phenotype CD44+CD90+CD105+CD73+CD45+CD31-CD34-CD45were used. All studied cultures did not express hematopoietic markers, but expressed the markers CD73, CD90, CD105, recommended by the International Society for Cellular Therapy to identify mesenchymal stromal cells. ${ }^{(14)}$

Using PCR, the presence of bacterial, viral and fungal contamination, including mycoplasmal, in dermal fibroblasts was determined. 
Determination of the area and condition of the postoperative scar

For the study and objectification of the surgical wound healing processes in patients of both groups, the area of scar formation was determined using the LesionMeter program for the Android operating system. To measure the surface area of the wound, a face or neck area with a scar was photographed, with a standard plastic bank card placed in view as a scaling standard. After that, on the received photo, a circle was drawn around the perimeter of the scar and its area was automatically obtained in $\mathrm{cm}^{2}$. The area of the healing wound was determined immediately after surgery, on Days 4, 7, 14, 21 and 30.

A physical examination of the healing wound was conducted to determine the nature of the healing and the color and density of the scar.

Thermometry of a trophic ulcer

To study the nature and extent of functional disorders in the wound area, their dynamics in the process of treatment and scar formation, we used contact liquid crystal thermography, which was carried out using thermo indicator films (CelluVision kit, IPS Italy), representing a layer of liquid crystal with a thickness of about 3 microns between two polymer films with a thickness of 5-10 microns each. The liquid crystal composition of the thermo indicator film is sealed around the perimeter; it works in the general temperature range from $34.1^{\circ} \mathrm{C}$ to $37.5^{\circ} \mathrm{C}$. The color palette (red-34.1-34. $2^{\circ} \mathrm{C}$, orange $-34.3-34.4^{\circ} \mathrm{C}$, yellow-34.5$34.7^{\circ} \mathrm{C}$, green $-34.8-35.0^{\circ} \mathrm{C}$, light blue $-35.1-35.4^{\circ} \mathrm{C}$, blue$35.5-35.8^{\circ} \mathrm{C}$, violet $-35.9-37.5^{\circ} \mathrm{C}$ ) allows acquisition of color transitions in the spectrum from red to blue with a temperature change of $1.5-2^{\circ} \mathrm{C}$; the threshold sensitivity of the color transition is $0.1{ }^{\circ} \mathrm{C}$. Conducting contact thermography allows evaluation of the individual characteristics of the distribution of temperature fields, to identify asymmetric areas of skin hypo- or hyperthermia, which are amenable to qualitative and quantitative analysis. The resulting color picture was examined visually and photographed. These thermograms were evaluated according to the following parameters: detection of thermal asymmetry, study of the area of the hypoor hyperthermia area (dimensions, degree of uniformity), and determination of the temperature gradient in the wound (rumen) and surrounding tissues.

Statistical analysis was performed using the statistical software «Statistica». (v6.0, StatSoft, USA) and Microsoft Excel 2007. Baseline characteristics were summarized as frequencies and percentages for categorical variables and as mean \pm SEM for continuous variables. The Mann-Whitney (U Test) was used to compare the differences between the two independent groups. The Wilcoxon criterion was used to compare the differences between the paired samples. A probability value of $P<0.05$ was considered statistically significant.

\section{Results}

On Day 1 after the operation, according to the results of planimetry, the area of the protective wound in both groups was the same and amounted to $1.5 \pm 0.04 \mathrm{~cm}^{2}$ (Table 2). On thermograms in the area of the seam line, a thin "cold line" was determined, which is a dark line in the section plane. It is surrounded by a zone of moderately high brightness in a radius of $2-5 \mathrm{~cm}$.

Table 2.

The dynamics of the healing of surgical wound (SW) in patients of Groups 1 and 2

\begin{tabular}{|l|c|c|c|c|}
\hline $\begin{array}{c}\text { Day of } \\
\text { observation }\end{array}$ & $\begin{array}{c}\text { Group 1 } \\
\text { (area of } \\
\left.\text { SW in } \mathrm{cm}^{2}\right)\end{array}$ & $\begin{array}{l}\text { Changes in SW } \\
\text { area in Group 1 } \\
\text { relative to the } \\
\text { previous } \\
\text { Day of obser- } \\
\text { vation, in \% }\end{array}$ & $\begin{array}{c}\text { Group 2 } \\
\left.\text { SW in } \mathrm{cm}^{2}\right)\end{array}$ & $\begin{array}{l}\text { Changes in SW } \\
\text { area in Group 1 } \\
\text { relative to the } \\
\text { previous } \\
\text { Day of obser- } \\
\text { vation, in \% }\end{array}$ \\
\hline 1 & $1.3 \pm 0.04$ & - & $1.3 \pm 0.04$ & - \\
\hline 3 & $0.95 \pm 0.06^{*}$ & -26.92 & $1.10 \pm 0.04$ & -13.64 \\
\hline 7 & $0.90 \pm 0.01^{*}$ & -5.30 & $1.00 \pm 0.01$ & -9.09 \\
\hline 14 & $0.78 \pm 0.04^{*}$ & -13.33 & $0.98 \pm 0.03$ & -2.00 \\
\hline 21 & $0.69 \pm 0.02^{*}$ & -11.54 & $0.87 \pm 0.03$ & -11.22 \\
\hline 30 & $0.42 \pm 0.05^{*}$ & -39.13 & $0.65 \pm 0.04$ & -25.29 \\
\hline
\end{tabular}

*- $P<0.05$ between Group 1 and Group 2.

When examining wounds on Day 3, we revealed a difference in the magnitude of postoperative swelling of the soft tissues. Infiltrates and hematomas were not marked. In Group 2, the swelling was visually more pronounced than in patients who underwent postoperative wound ablation with xenogenic fibroblasts. The stitches were retained, however, due to pronounced postoperative edema as a result of the eruption of the skin with a suture thread; in 3 patients of Group 2 we found a partial divergence of the wound edges. The gaping area of the wound was from 1 to $3 \mathrm{~mm}$ in length and from 1 to $2 \mathrm{~mm}$ in width. There was a slight hyperemia of the wound edges, its size was $1.5 \pm 0.05 \mathrm{~cm}(P<0.05)$. The wound area in patients of Group 1 for three days after surgery decreased more than in Group 2 (Table 2), and was statistically significantly lower by $15.38 \%$.

The data of the thermographic study on Day 3 after the operation showed that in both groups the area of bright blue-violet luminescence (hyperthermia) corresponded in size to the clinically determined sizes of postoperative edema (Fig.1). The contours of the hyperthermia zone had no clear boundaries, and smoothly and diffusely passed into the region of less intense luminescence. During this period, there was a significant variation in the temperature gradient between the wound and the surrounding tissues: in Group 1 it was $0.4-0.8^{\circ} \mathrm{C}$, in Group 2 it was $0.6-1.0^{\circ} \mathrm{C}$.

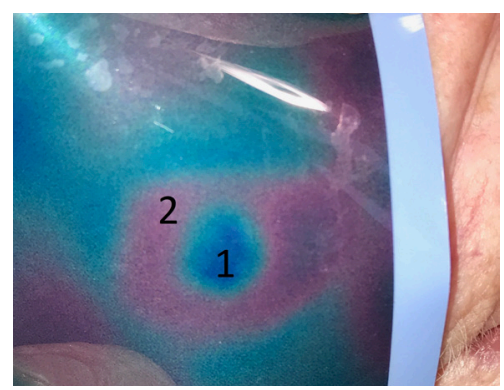

Fig. 1. Thermographic picture of the scar of Patient B. on Day 3 after surgery. 1- hyperthermia zone; 2 - a zone of moderate temperature. 
Changes in digital values during thermography objectively reflect the dynamics and nature of local inflammatory processes and circulatory disorders in regenerating wounds. The analysis of the data we obtained on Day 3 indicates the presence of inflammatory changes and circulatory disorders in the wound, which are most pronounced in patients of Group 2. The use of fibroblasts has significantly reduced the severity of inflammatory processes in patients of Group 1.

By Day 7 after surgery, the area of wounds in patients of both groups was reduced, but the area of wounds in Group 1 was $11.11 \%$ less than in Group 2. Wound healing occurred by primary intention in patients of both groups. Postoperative edema was practically absent in Group 1 (Fig.2). In patients in Group 2, there remained mild hyperemia of the edges of wounds; the size of the wound averaged $0.3 \pm 0.02 \mathrm{~cm}(P<0.05)$.

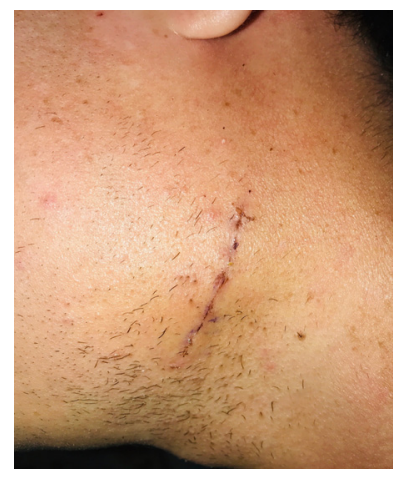

A

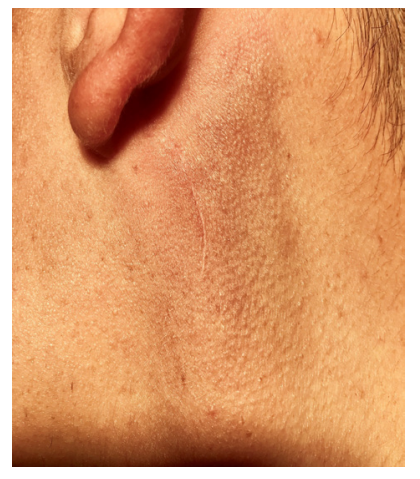

B
Fig. 2. Scar of Patient K. after surgery and transplantation of XDFs. A - Day 7; B - Day 30, formed normotrophic scar.

Thermographic studies conducted during this period revealed the presence of thermal asymmetry of the operated and healthy sides, which manifested itself in a somewhat expanded luminescence zone in the area of the operation. In general, in the thermograms of patients in both groups, the heterogeneity of the structure of the operated area remained, and the temperature gradient was $0.2-0.4^{\circ} \mathrm{C}$. Only in 3 patients of Group 2, in which the wound edges diverged on Day 3, did the hyperthermia reaction remain in the wound area; the temperature gradient was $0.6-0.8^{\circ} \mathrm{C}$.

Analysis of the wound healing results allows us to state that after excision of skin tumors, the introduction of a cell culture of XDFs stimulates and positively influences the healing processes, which was manifested by the absence of edema and inflammatory phenomena in the wound edges, compared with the control group and proved by thermograms.

All patients on Day 7 after surgery had their stitches removed and daily tissue massage was prescribed.

Subsequently, on Days 14 and 21, patients of both groups underwent a routine examination, and the forming scars were measured. Table 2 presents the results of measurements, showing a gradual decrease in the area of scars, statistically significantly greater in patients of Group 1 (by $20.41 \%$ and $20.69 \%$, respectively).

On Day 30 after the operation, the area of scars in patients of Group 1 had decreased more significantly than in
Group 2; the difference between them was $35.38 \%$. In addition to routine inspection, the geometric characteristics of the young scar were determined. Three grades were distinguished by width: thin - up to $1 \mathrm{~mm}$, medium - up to $2 \mathrm{~mm}$, and wide - over $2 \mathrm{~mm}$.

In Group 1, 10/71.43\% patients had thin scars, $4 / 28.57 \%$ had medium scars, and wide scars were not recorded. During palpation, scars were soft and mobile, on the same level with intact skin (defined as normotrophic). In Group 2, 5/41.67\% patients had thin scars, $5 / 41.67 \%$ had medium scars and wide scars were recorded in 2 2/16.67\% patients. In Group 2, during palpation, medium and wide scars were somewhat denser, and in wide scars with the underlying tissues, lumpiness and soreness remained. At the same time, thin and medium scars were normotrophic, and wide ones, which we identified as hypotrophic, were 1-1.2 $\mathrm{mm}$ below the level of intact skin. The fact that the scars in patients of Group 1 were a lighter color by this time drew attention. In Group 2, the scars had a reddish or bluish tint.

The thermographic picture on Day 30 in patients of Group 1 with thin scars revealed the absence of thermal asymmetry with the opposite side. The structure of the operated zone was homogeneous. Consequently, microcirculation was fully restored in the formed scar. In cases of medium-wide scars, by this time a thin (1-2 mm) heterogeneous (intermittent) band of hypothermia (cold zone of red color) was revealed, indicating weak vascularization, which gradually turned into a glow characteristic of healthy tissues. Thermograms in Group 2 were different. In patients with thin and medium-wide scars, in all cases a thin band of hypothermia (cold zone of red color) was determined, corresponding to the width of the scar, surrounded by a zone of brighter green-violet luminescence, indicating a local slight hyperthermia, probably due to the inflammatory response that was not completely overcome (Fig.3) . The zone of small hyperthermia smoothly passed into the zone of intact surrounding tissues. Thermal asymmetry with the opposite side was noted. In 2 patients with wide scars on the thermograms, a band of hypothermia was distinguished, which was somewhat wider than the scar that was visually detected on the skin. A small hyperthermia zone remained around the scar, which diffusely passed into the thermal zone of intact surrounding tissues. The temperature gradient was $0.2-0.4^{\circ} \mathrm{C}$.

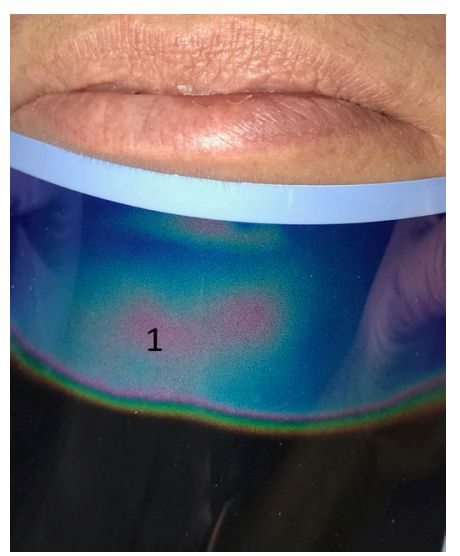

Fig. 3. Thermographic picture of the average width of the cicatrix of Patient S. from Group 2 on Day 30 after surgery. 1- a hypothermia zone. 


\section{Discussion}

The progression of wound healing, including skin, is called the wound process and includes three stages: inflammation, proliferation with the formation of granulation tissue, and remodeling or fibrosis. ${ }^{(15)}$ Wound healing without scarring is possible only in fetuses in the first 6-7 months of pregnancy due to the absence of the inflammatory phase of the wound process. ${ }^{(16)}$ It is inflammation that plays a key role in the morphology of the future scar. In normal wound healing, inflammatory cells flow to the wound site up to Day 4-6, followed by the proliferative phase, during which the inflammatory cells are replaced by fibroblasts. The transition between inflammation and proliferation is important because abnormal inflammatory prolongation leads to excessive scarring. ${ }^{(17)}$ Since uncontrolled and prolonged inflammation of the dermis causes pathological scarring, a decrease in inflammation and faster healing of wounds has a beneficial effect on the formation of normotrophic scars. ${ }^{(18)}$ Continuous and localized inflammation of granulation tissue causes pathological scars, primarily hypertrophic scars. ${ }^{(18)}$ If an abnormal fibroblast reaction to inflammation is present, a keloid scar is formed. ${ }^{(19)}$

Evidence accumulated in recent years suggests that MSCs contribute to the healing of wounds without scars and prevent the formation of fibrous tissue, which makes them potentially effective for the formation of a normotrophic scars that are hardly noticeable, which is highly desirable in the head and neck. ${ }^{(20)}$ However, the search for means to reduce the duration of inflammation revealed that human dermal fibroblasts are the source of adiponectin, which acts as an active anti-inflammatory cytokine and induces the production of anti-inflammatory factors, such as IL-10 and IL-1RA. ${ }^{(21)}$ In addition, fibroblasts, unlike MSCs, are not oncogenic and have low expression of histocompatibility antigens, which allows the use of populations of both auto and xenofibroblasts. ${ }^{(10)}$ Experimental studies in our laboratory found that fibroblasts have a high ability to shorten the healing time and increase the vascularization of wounds, significantly improving the characteristics of the formed scars. ${ }^{(22)}$

Studies by a number of authors have found that the total number of fibroblasts and their proliferative capacity in the dermis of the skin of an adult are relatively small and progressively decrease with age. ${ }^{(23)}$ Introduction to the primary wound of a sufficient number of active fibroblasts - which naturally require up to 3-4 days for migration, proliferation and transformation to start the formation of granulation tissue - reduces the healing time and changes the characteristics of the scar for the better. We found that within 30 days after surgery, the area of the wound and scar during transplantation of XDFs decreased by $67.69 \%$, while without fibroblast transplantation, the area decreased by only $50.0 \%$. In addition, $71.43 \%$ of patients in Group 1 (versus $41.67 \%$ of patients in the control group) had thin soft normotrophic scars, in which the microcirculation was restored by thermography, indistinguishable from the surrounding skin and the skin of symmetrical areas of the face and neck.

The informativeness of the color thermographic method is recognized by many authors, since it reliably reflects the dynamics of the wound process, which makes it possible to judge the nature of metabolic shifts at any stage of wound healing. ${ }^{(24,25)}$ Such a study did not require special equipment or wasted time, and did not harm patients, did not violate the physiological processes in their body and did not cause inconvenience. It attracted attention to the elevated temperature around the periphery of medium-wide and wide scars that remained in control group patients on Day 30, indicating a weak inflammatory response. In this case, patients were advised to continue monitoring with the attending physician.

\section{Conclusion}

The analysis of the parameters of the young scar formation on Day 30 after the operation makes it possible to state that the introduction of cell culture of XDFs in the wound edges has a positive effect on the healing rate of surgical wounds, decreases the inflammatory response and contributes to the development of a distinctively positive scar in terms of its quality and functional characteristics. By Day 30, the primary surgical wound area was reduced by $67.69 \%$, and $71.43 \%$ of patients had soft and thin aesthetic scars with microcirculation that were not distinguishable from the surrounding skin and the skin of symmetrical areas of the face or neck. In patients of the control group, without fibroblast transplantation, the area decreased by only $50.0 \%$ and aesthetic scars were formed only in $41.67 \%$ of cases. In $16.67 \%$ of patients, the presence of wide, dense, cold (due to weak vascularization) hypotrophic scars was noted. Hyperthermia persists around these scars, indicating a weak inflammatory response.

Thus, the use of a cell culture suspension of XDFs in the treatment of postoperative surgical wounds opens up new real possibilities for reducing the incidence of inflammatory reactions, stimulates healing processes and contributes to the development of more functionally and aesthetically acceptable scars on the face and neck.

\section{Competing Interests} interests.

The authors declare that they have no competing

\section{References}

1. Broughton G, Janis JE, Attinger CE. The basic science of wound healing. Plast Reconstr Surg. 2006;117(7 Suppl):12S-34S. 2. Profyris C, Tziotzios C, Do Vale I. Cutaneous scarring: Pathophysiology, molecular mechanisms, and scar reduction therapeutics Part I. The molecular basis of scar formation. J Am Acad Dermatol. 2012;66(1):1-10. doi: 10.1016/j. jaad.2011.05.055.

3. Bielefeld KA, Amini-Nik S, Alman BA. Cutaneous wound healing: recruiting developmental pathways for regeneration. Cell Mol Life Sci. 2013;70(12):2059-81. doi: 10.1007/s00018-012-1152-9.

4. Atiyeh BS, Amm CA, El Musa KA. Improved scar quality following primary and secondary healing of cutaneous wounds. Aesthetic Plast Surg. 2003;27(5):411-7.

5. Belousov AE. Scars and their correction. SPb.: 
"Commander-SPB"; 2005. [In Russian].

6. Bock O, Schmid-Ott G, Malewski P, Mrowietz U. Quality of life of patients with keloid and hypertrophic scarring. Arch Dermatol Res. 2006;297(10):433-8.

7. Mofikoya BO, Adeyemo WL, Ugburo AO. An overview of biological basis of pathologic scarring. Niger Postgrad Med J. 2012;19(1):40-45.

8. Agarwal C, Britton ZT, Alaseirlis DA, Li Y, Wang JHC. Healing and normal fibroblasts exhibit differential proliferation, collagen production, alpha-SMA expression, and contraction. Ann Biomed Eng. 2006;34(4):653-9.

9. Carantino I, Florescu IP, Carantino A. Overview about the keloid scars and the elaboration of a non-invasive, unconventional treatment. J Med Life. 2010;3(2):122-7.

10. Kolokol'tseva TD, Iurcnenko ND, Kolosov NG, Nechaeva EA, Shumakova OV, Khrtisto SA. [Prospects of use human fetal fibroblasts in the treatment of various etiology wounds]. Vestn Ross Akad Med Nauk. 1998;(3):32-5. [Article in Russian].

11. Fang F, Huang RL, Zheng Y, Liu M, Huo R. Bone marrow derived mesenchymal stem cells inhibit the proliferative and profibrotic phenotype of hypertrophic scar fibroblasts and keloid fibroblasts through paracrine signaling. J Dermatol Sci. 2016;83(2):95-105. doi: 10.1016/j.jdermsci.2016.03.003. 12. Namazi MR, Fallahzadeh MK, Schwartz RA. Strategies for prevention of scars: what can we learn from fetal skin? Int J Dermatol. 2011;50(1):85-93. doi: 10.1111/j.13654632.2010.04678.x.

13. Li H, Fu X. Mechanisms of action of mesenchymal stem cells in cutaneous wound repair and regeneration. Cell Tissue Res. 2012;348(3):371-7. doi: 10.1007/s00441-012-1393-9.

14. Lupatov AU, Vdovin AS, Vakhrushev IW, Poltavtseva RA, Yarygin KN. [Comparative analysis of the expression of surface markers on fibroblasts and fibroblast-like cells isolated from various human tissues]. Cell Technologies in Biology and Medicine. 2014;4:221-228. [Article in Russian].

15. Luo L, Li J, Liu H, Jian X, Zou Q, Zhao Q, et al. Adiponectin is involved in connective tissue growth factor- induced proliferation, migration and overproduction of the extracellular matrix in keloid fibroblasts. Int J Mol Sci. 2017; 18(5). pii: E1044. doi: 10.3390/ijms18051044.

16. Wilgus TA. Regenerative healing in fetal skin: a review of the literature. Ostomy Wound Manage. 2007;53(6):16-31.

17. Walmsley GG, Maan ZN, Hu MS, Atashroo DA, Whittam AJ, Duscher D, et al. Murine dermal fibroblast isolation by FACS. J Vis Exp. 2016;(107):53430. doi: 10.3791/53430.

18. Ogawa R. Surgery for scar revision and reduction: from primary closure to flap surgery. Burns Trauma. 2019;7:7. doi: 10.1186/s41038-019-0144-5.

19. Seo BF, Jung SN. The immunomodulatory effects of mesenchymal stem cells in prevention or treatment of excessive scars. Stem Cells Int. 2016;2016:6937976. doi: $10.1155 / 2016 / 6937976$.

20. Liu J, Ren J, Su L, Cheng S, Zhou J, Ye X, et al. Human adipose tissue-derived stem cells inhibit the activity of keloid fibroblasts and fibrosis in a keloid model by paracrine signaling. Burns. 2018;44(2):370-385. doi: 10.1016/j.burns.2017.08.017.

21. Esfahani M, Movahedian A, Baranchi M. Adiponectin: an adipokine with protective features against metabolic syndrome. Iran J Basic Med Sci. 2015;18(5):430-42.

22. Shapovalova YeYu, Boyko TA, Baranovskiy YuG, Morozova MN, Barsukov NP, Ilchenko FN, et al. Effects of fibroblast transplantation on the content of macrophages and the morphology of regenerating ischemic cutaneous wounds. International Journal of Biomedicine. 2017;7(4):302-306. doi. org/10.21103/Article7(4)_OA6.

23. Petrov VV. [Age-related changes in the number of CD45+ cells in human dermis]. Adv Gerontol. 2012;25(4):598-603. [Article in Russian].

24. Itoh Y, Arai K. Use of recovery-enhanced thermography to localize cutaneous perforators. Ann Plast Surg. 1995;34(5):507-11.

25. Renkielska A, Nowakowski A, Kaczmarek M, Dobke MK, Grudziński J, Karmolinski A, et al. Static thermography revisited - an adjunct method for determining the depth of the burn injury. Burns. 2005;31(6):768-75. 